STRUCTURAL BIOLOGY

ISSN 2059-7983

Keywords: FMN-dependent monooxygenase; protein structure; industrial biocatalysis; corrigendum

\section{The oxygenating constituent of 3,6-diketocamphane monooxygenase from the CAM plasmid of Pseudomonas putida: the first crystal structure of a type II Baeyer-Villiger monooxygenase. Corrigendum}

\author{
Michail N. Isupov, ${ }^{a}$ Ewald Schröder, ${ }^{a}$ Robert P. Gibson, ${ }^{a}$ Jean Beecher, ${ }^{a}$ Giuliana \\ Donadio, ${ }^{a}$ Vahid Saneei,, ${ }^{a}$ Stephlina A. Dcunha, ${ }^{a}$ Emma J. McGhie, ${ }^{a}$ Christopher \\ Sayer, ${ }^{a}$ Colin F. Davenport, ${ }^{\text {a }}$ Peter C. K. Lau, ${ }^{\text {b }}$ Yoshie Hasegawa, ${ }^{\text {c }}$ Hiroaki Iwaki, ${ }^{c}$ \\ Maria Kadow, ${ }^{d}$ Kathleen Balke, ${ }^{d}$ Uwe T. Bornscheuer, ${ }^{d}$ Gleb Bourenkov ${ }^{\mathbf{e}}$ and \\ Jennifer A. Littlechild ${ }^{\mathrm{a} *}$
}

\begin{abstract}
a The Henry Wellcome Building for Biocatalysis, Biosciences, College of Life and Environmental Sciences, University of Exeter, Stocker Road, Exeter EX4 4QD, England, ${ }^{\mathbf{b}}$ Biotechnology Research Institute, National Research Council Canada, 6100 Royalmount Avenue, Montreal, QC H4P 2R2, Canada, 'Department of Biotechnology, Faculty of Engineering, Kansai University, Japan, ${ }^{\mathbf{d}}$ Department of Biotechnology and Enzyme Catalysis, Institute of Biochemistry, Greifswald University, Felix-Hausdorff-Strasse 4, 17487 Greifswald, Germany, and ${ }^{\mathbf{e} E u r o p e a n ~ M o l e c u l a r ~ B i o l o g y ~ L a b o r a t o r y ~(E M B L), ~}$ Hamburg Outstation, Notkestrasse 85, 22607 Hamburg, Germany. *Correspondence e-mail: j.a.littlechild@exeter.ac.uk
\end{abstract}

A statement is amended in the article by Isupov et al. [(2015). Acta Cryst. D71, 2344-2353].

In the article by Isupov et al. (2015), the statement in $\$ 3.10$ 'Partial sequencing of the large CAM plasmid has now identified a flavin reductase adjacent to the 3,6-DKMO gene on the CAM plasmid (Littlechild \& Isupov, unpublished data)' is withdrawn. The following statement is added: 'A cognate flavin reductase (Fred) from the genome of Pseudomonas putida (strain PpCam), which provides the required FMNH2 for the 3,6-DKMO activity, has been described by Iwaki et al. (2013). Other non-cognate reductases have been reported which can partially support the DKMO activity but not as efficiently as the cognate Fred'.

\section{References}

Isupov, M. N., Schröder, E., Gibson, R. P., Beecher, J., Donadio, G., Saneei, V., Dcunha, S. A., McGhie, E. J., Sayer, C., Davenport, C. F., Lau, P. C., Hasegawa, Y., Iwaki, H., Kadow, M., Balke, K., Bornscheuer, U. T., Bourenkov, G. \& Littlechild, J. A. (2015). Acta Cryst. D71, 2344-2353.

Iwaki, H., Grosse, S., Bergeron, H., Leisch, H., Morley, K., Hasegawa, Y. \& Lau, P. C. K. (2013). Appl. Environ. Microbiol. 79, 3282-3293.

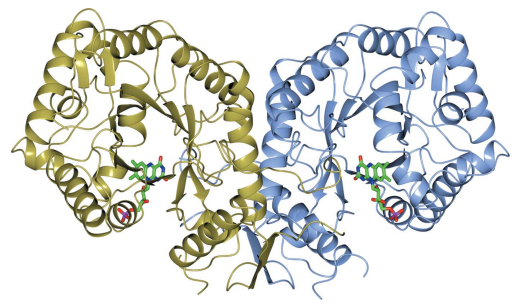

(C) 2018 International Union of Crystallography 
BIOLOGICAL CRYSTALLOGRAPHY

ISSN 1399-0047

Received 22 June 2015

Accepted 24 September 2015

Edited by M. Schiltz, Fonds National de la Recherche, Luxembourg

Keywords: FMN-dependent monooxygenase; protein structure; industrial biocatalysis.

PDB references: type II Baeyer-Villiger monooxygenase, apo, 5aec; FMN complex, 4uwm

Supporting information: this article has supporting information at journals.iucr.org/d

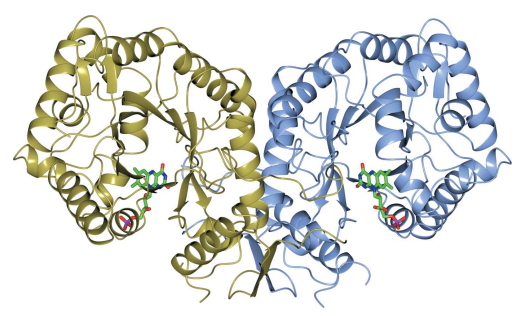

OPEN $\odot$ ACCESS

\section{The oxygenating constituent of 3,6-diketocamphane monooxygenase from the CAM plasmid of Pseudomonas putida: the first crystal structure of a type II Baeyer-Villiger monooxygenase}

\author{
Michail N. Isupov, ${ }^{a}$ Ewald Schröder, ${ }^{\text {a }}$ Robert P. Gibson, ${ }^{a}$ Jean Beecher, ${ }^{a}$ Giuliana \\ Donadio, ${ }^{a}$ Vahid Saneei, ${ }^{a}$ Stephlina A. Dcunha, ${ }^{a}$ Emma J. McGhie, ${ }^{a}$ Christopher \\ Sayer, ${ }^{\mathrm{a}}$ Colin F. Davenport, ${ }^{\mathrm{a}}$ Peter C. Lau, ${ }^{\mathrm{b}}$ Yoshie Hasegawa, ${ }^{\mathrm{c}}$ Hiroaki Iwaki, \\ Maria Kadow, ${ }^{d}$ Kathleen Balke, ${ }^{d}$ Uwe T. Bornscheuer, ${ }^{d}$ Gleb Bourenkov ${ }^{e}$ and \\ Jennifer A. Littlechild ${ }^{\mathrm{a} *}$
}

\begin{abstract}
a The Henry Wellcome Building for Biocatalysis, Biosciences, College of Life and Environmental Sciences, University of Exeter, Stocker Road, Exeter EX4 4QD, England, biotechnology Research Institute, National Research Council Canada, 6100 Royalmount Avenue, Montreal, QC H4P 2R2, Canada, 'Department of Biotechnology, Faculty of Engineering, Kansai University, Japan, dDepartment of Biotechnology and Enzyme Catalysis, Institute of Biochemistry, Greifswald University, Felix-Hausdorff-Strasse 4, 17487 Greifswald, Germany, and ${ }^{\mathbf{e} E u r o p e a n ~ M o l e c u l a r ~ B i o l o g y ~ L a b o r a t o r y ~(E M B L), ~}$ Hamburg Outstation, Notkestrasse 85, 22607 Hamburg, Germany. *Correspondence e-mail: j.a.littlechild@exeter.ac.uk
\end{abstract}

The three-dimensional structures of the native enzyme and the FMN complex of the overexpressed form of the oxygenating component of the type II BaeyerVilliger 3,6-diketocamphane monooxygenase have been determined to $1.9 \AA$ resolution. The structure of this dimeric FMN-dependent enzyme, which is encoded on the large CAM plasmid of Pseudomonas putida, has been solved by a combination of multiple anomalous dispersion from a bromine crystal soak and molecular replacement using a bacterial luciferase model. The orientation of the isoalloxazine ring of the FMN cofactor in the active site of this TIM-barrel fold enzyme differs significantly from that previously observed in enzymes of the bacterial luciferase-like superfamily. The Ala77 residue is in a cis conformation and forms a $\beta$-bulge at the $\mathrm{C}$-terminus of $\beta$-strand 3 , which is a feature observed in many proteins of this superfamily.

\section{Introduction}

The use of enzymes in commercial biocatalysis is of increasing importance for the fine-chemical and pharmaceutical industries, with the number of new drugs produced in this way due to increase dramatically over the next few years (Wohlgemuth, 2010; Bornscheuer et al., 2012; Reetz, 2013). The application of enzymes has considerable advantages in that the process is environmentally friendly and enzymes have the ability to produce single enantiomeric products, which are of value as chiral building blocks for new drug molecules (Lilly et al., 1996; Littlechild et al., 2007).

The Baeyer-Villiger oxidation reaction (Baeyer \& Villiger, 1899) is an important reaction for synthetic organic chemistry in which linear/cyclic ketones react with peroxy acids to produce a corresponding ester/lactone. The application of the Baeyer-Villiger reaction for the chemical synthesis of antibiotics, steroids and pheromones has been reviewed by Krow (1993). The purity and stereospecificity of the reaction products required by the pharmaceutical industry is difficult to achieve by traditional chemical processes owing to the high reactivity of peracids (Roberts \& Wan, 1998). The use of peracids and solvents on a large scale is not environmentally 
friendly. Therefore, there is increasing interest from industry in enzymes that can carry out a Baeyer-Villiger oxidation reaction (Alphand et al., 2003).

Several microorganisms can produce $\mathrm{NAD}(\mathrm{P}) \mathrm{H}$-dependent flavoenzymes capable of catalyzing Baeyer-Villiger reactions (Leisch et al., 2011; Balke et al., 2012), which use a stable flavin-peroxide intermediate to attack the carbonyl $\mathrm{C}$ atom of the substrate in a reaction identical to the equivalent nonenzymatic reaction. These enzymes are called Baeyer-Villiger monooxygenases (BVMOs) and are classified into two main types depending on the flavin cofactor. The more studied type I BVMOs are proteins utilizing the FAD cofactor, which is reduced by an NADPH bound to the same polypeptide chain. The first type I BVMO crystal structure was reported for the phenylacetone monooxygenase from Thermobifida fusca (Malito et al., 2004). Type II BVMOs utilize a FMN cofactor, which is reduced by a separate NADH dehydrogenase enzyme. These enzymes have been relatively understudied compared with the type I enzymes owing to their more complex multi-enzyme structure. The substrate specificity of type II BVMOs is different from the type I enzymes and they are increasingly important for commercial biocatalysis since they have the ability to oxidize bicyclic lactones (Alphand $e t$ al., 2003) and because they utilize the cheaper cofactor NADH.

It has been known for some time that the bacterium Pseudomonas putida NCIMB 10007 is able to grow on either enantiomer of the natural bicyclic monoterpene camphor as the sole carbon source (LeGall et al., 1963). The oxidative degradation pathway of camphor requires two enantiocomplementary type II BMVOs (Conrad et al., 1965), 2,5-diketocamphane 1,2-monooxygenase (2,5-DKMO; EC 1.14.13.162) and 3,6-diketocamphane 1,6-monooxygenase (3,6-DKMO; EC 1.14.13.-), which catalyse the NADH-dependent lactonization of diketocamphane stereoisomers (2,5- and 3,6-diketocamphane). The latter are intermediates in the oxidative degradation pathway of (+)- and (-)-camphor, respectively (Fig. 1). Both DKMOs are located on the large CAM plasmid harboured in P. putida and are induced when either racemic camphor or one of its stereoisomers are present in the growth medium (Shaham et al., 1973). The DKMO enzymes have been reported to be built up from a dimeric oxygenating component and a loosely associated NADH dehydrogenase (Jones et al., 1993). Both the 2,5-DKMO and 3,6-DKMO oxygenating components have sequence similarity to bacterial luciferases (McGhie, 1998) and bear little similarity to type I BVMOs. The molecular masses of the 2,5-DKMO and 3,6-DKMO monomers are 40.7 and $42.3 \mathrm{kDa}$, respectively (McGhie et al., 1998).

The two DKMO enzymes have been shown to carry out oxygenation reactions with a variety of natural ketones and their synthetic analogues using partially purified preparations of the wild-type enzyme (Grogan, Roberts \& Willetts, 1993; Grogan, Roberts, Wan et al., 1993; Grogan et al., 1994; Gagnon et al., 1994, 1995), where a mixture of both DKMOs was called MO1. This preparation was also able to utilize a range of aryl alkyl and alkyl alkyl sulfides. The two DKMOs can use natural and synthetic ketones with distinct enantioselectivity and they have been exploited in key reactions for the chemoenzymatic synthesis of commercially useful products (Grogan et al., 1992; Grogan, Roberts \& Willetts, 1993; Grogan, Roberts, Wan et al., 1993; Gagnon et al., 1994, 1995). The results of the different ketone and sulfide biotransformations were used to produce simple models for the substrate-binding pockets of both enzymes, which implied that there were differences between them (Beecher \& Willetts, 1998).

The mechanism of enantioselectivity for the hydroxylperoxide rearrangement taking place in the BVMO active site

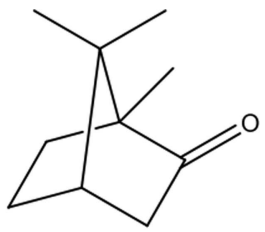

(-)-Camphor

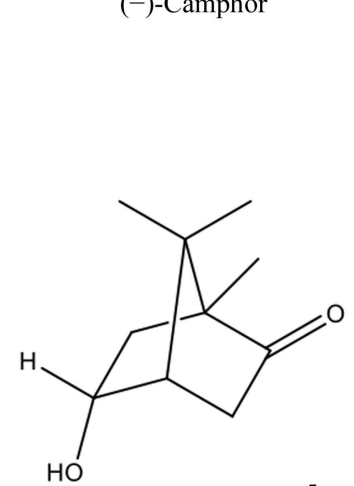

5-exo-Hydroxycamphor

(1)

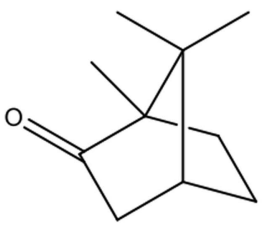

(+)-Camphor

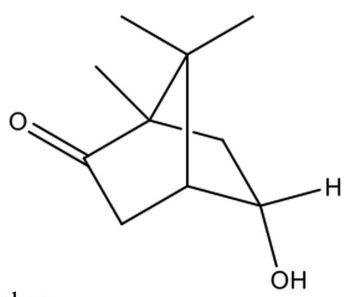

(2)

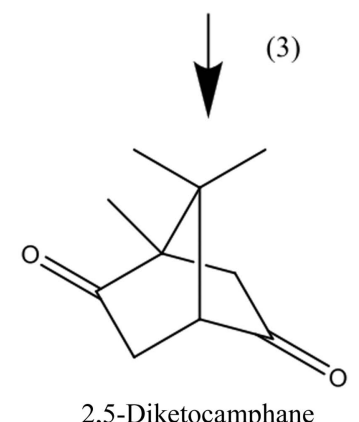

3,6-Diketocamphane

2,5-Diketocamphane
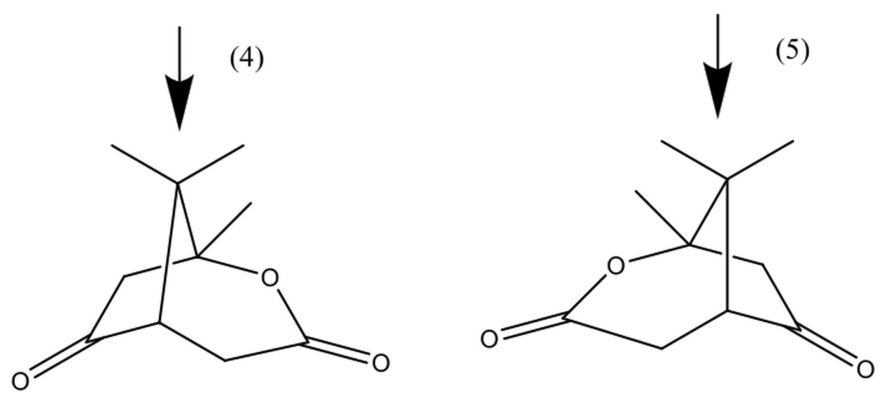

Unstable lactone products

Figure 1

The camphor-degradation pathway (adapted from McGhie et al., 1998). The following enzymes are indicated: (1) 5-exo-hydroxylase (P450 CAM), (2) and (3) exo-hydroxycamphor dehydrogenases, (4) 3,6-DKMO, (5) 2,5-DKMO. 
was proposed by Kelly (1996) to be owing to diastereofacial selection by the flavin cofactor. Type II BVMOs were attributed to the $\mathrm{R}$ group in relation to the $r e$-face attachment of the hydroperoxide to the flavin coenzyme.

Initial structural characterization studies of 3,6-DKMO by our group were with the native enzyme purified from camphor-induced $P$. putida cell cultures. This was the only isozyme able to be purified in sufficient quantities for crystallization (McGhie et al., 1998). Only preliminary structural studies have been reported by us to date for the type II enzymes (McGhie et al., 1998; Isupov \& Lebedev, 2008). Detailed structural and mechanistic comparisons of the two DKMO isozymes, which have $45 \%$ sequence identity (Kadow et al., 2012), and knowledge of their active sites will enable rationalization of their substrate specificity and their potential optimization by site-directed mutagenesis to use new industrially relevant substrates.

Multiple attempts to overexpress 3,6-DKMO in Escherichia coli were unsuccessful until the application of the Takara chaperone system (Kadow et al., 2012). Using 3,6-DKMO produced using this expression system has allowed further protein crystals to be grown of the oxygenating subunit in both the apo form and in the presence of the weakly bound FMN cofactor. This has allowed the location of the bound cofactor and a comparison between the native enzyme and cofactorcomplex structures to be carried out.

\section{Experimental}

\subsection{Materials}

All reagents were obtained from Sigma-Aldrich, Buchs, Switzerland unless otherwise stated. The chromatography columns were obtained from GE Healthcare (Little Chalfont, England).

\subsection{Enzyme purification}

The original purification of the native 3,6-DKMO from 201 batches of camphor-induced cells of P. putida NCIMB 10007 was reported by us previously (McGhie et al., 1998; McGhie \& Littlechild, 1996). The cells were harvested by centrifugation and disrupted by sonication. After the removal of cell debris, an ammonium sulfate precipitation step was followed by dialysis in buffer $A\left[20 \mathrm{~m} M \mathrm{KH}_{2} \mathrm{PO}_{4} / \mathrm{K}_{2} \mathrm{HPO}_{4} \mathrm{pH} 7.1,6 \mathrm{~m} M\right.$ $\beta$-mercaptoethanol, $0.1 \mathrm{~m} M$ EDTA, $10 \mu M$ phenylmethylsulfonylfluoride (PMSF), $20 \mu M$ benzamidine (BAM)]. The protein was applied onto a Fast Flow $Q$ anion-exchange chromatography column and eluted with a linear gradient of $0-0.45 M$ potassium chloride in buffer $A$. After dialysis in buffer $A$, the protein was applied onto a Mono $\mathrm{Q}$ anionexchange column and eluted with a linear gradient of 0 $0.45 M$ potassium chloride in buffer $A$. This was followed by size-exclusion chromatography in buffer $A$ containing $5 \%$ ammonium sulfate using a Superose 12 gel-filtration column. The fractions containing native 3,6-DKMO were monitored by NADH consumption at $340 \mathrm{~nm}$ and by analysis of the products of the reaction by gas chromatography (McGhie et al., 1998).
Recombinant 3,6-DKMO was purified from E. coli BL21 (DE3) cells (Novagen, Darmstadt, Germany) harbouring the pET-28b (Novagen) vector with the 3,6-DKMO gene and the plasmid pGro7 of the Takara Chaperone Plasmid Set (Takara, Saint-Germain-en-Laye, France) grown in LB medium containing $30 \mu \mathrm{g} \mathrm{ml}^{-1}$ kanamycin and $30 \mu \mathrm{g} \mathrm{ml}^{-1}$ chloramphenicol at $37^{\circ} \mathrm{C}$ to an optical density at $600 \mathrm{~nm}$ of $0.5-0.6$ (Kadow et al., 2012). The culture was transferred to $20^{\circ} \mathrm{C}$ and the chaperones were induced by the addition of $2 \mathrm{mg} \mathrm{ml}^{-1}$ $\mathrm{L}$-arabinose and incubated for $30 \mathrm{~min}$. Protein expression was induced with $1 \mathrm{~m} M$ isopropyl $\beta$-D-1-thiogalactopyranoside for $4 \mathrm{~h}$ at $20^{\circ} \mathrm{C}$. The cells were harvested by centrifugation at $20000 \mathrm{~g}$. The cell paste from a 21 culture was resuspended in buffer $B\left(20 \mathrm{~m} M \mathrm{KH}_{2} \mathrm{PO}_{4} / \mathrm{K}_{2} \mathrm{HPO}_{4} \mathrm{pH}\right.$ 7.1, $6 \mathrm{~m} M \beta$-mercaptoethanol, $0.1 \mathrm{~m} M$ EDTA, $10 \mu M$ PMSF) at a concentration of $10 \%(w / v)$. Sonication was carried out using a Soniprep 150 (MSE UK Ltd, Lower Sydenham, England) followed by centrifugation at $20000 \mathrm{~g}$ to remove precipitated protein and cell debris. The $\mathrm{N}$-terminally hexahistidine-tagged protein was purified on a HiLoad nickel column using a linear gradient of $0-1 M$ imidazole in buffer $B$. The enzyme was further purified by gel filtration on a Superdex 200 gel-filtration column using buffer $B$ containing $0.1 M \mathrm{NaCl}$.

\subsection{Crystallization}

Crystals of native 3,6-DKMO were grown at room temperature by the vapour-diffusion technique using purified protein at $10 \mathrm{mg} \mathrm{ml}^{-1}$ mixed in equal volumes with $50 \mathrm{mM}$ PIPES pH 6.5, 50\% ammonium sulfate (McGhie et al., 1998). These crystals were flash-cooled in liquid $\mathrm{N}_{2}$ after a short soak in a cryoprotectant consisting of $30 \%(v / v)$ glycerol, $50 \mathrm{mM}$ PIPES pH 6.5, 55\% ammonium sulfate.

The overexpressed 3,6-DKMO protein was concentrated using a $10 \mathrm{kDa}$ membrane Vivaspin (Vivascience, Massachusetts, USA). Microbatch crystallization trials were set up with an Oryx 6 crystallization robot (Douglas Instruments, Hungerford, England) using the Morpheus and JCSG+ screens (Molecular Dimensions, Newmarket, England) and protein solution at $7 \mathrm{mg} \mathrm{ml}^{-1}$ containing $20 \mathrm{mM}$ FMN, $5 \mathrm{mM}$ NADH and $5 \mathrm{~m} M(-)$-camphor. The droplet consisted of a 50:50 ratio of protein solution to precipitant ratio and was covered with a 50:50 mixture of silicone and paraffin oils. The best crystals were flash-cooled in liquid $\mathrm{N}_{2}$ after a $30 \mathrm{~s}$ soak in a cryoprotectant consisting of $100 \mathrm{~m} M$ HEPES $\mathrm{pH} 7.0$, $30 \%(v / v)$ PEG 200, 16\% PEG 3350, $100 \mathrm{mM} \mathrm{NaCl}, 20 \mathrm{~m} M$ FMN.

\subsection{Data collection}

The native 3,6-DKMO data were collected to $1.9 \AA$ resolution (Table 1) at $100 \mathrm{~K}$ at station BW7B at the EMBL Outstation at DESY, Hamburg, Germany using a MAR345 image-plate detector (MAR Research, Norderstedt, Germany). Three-wavelength MAD data sets were collected at the $\mathrm{Br} K$ edge for the crystal of the native enzyme, which was soaked for $1 \mathrm{~min}$ in a cryoprotectant consisting of $1 \mathrm{M}$ $\mathrm{NaBr}$ for $\mathrm{Br}$ anomalous phasing (Dauter et al., 2000). These 
Table 1

Summary of data-collection, phasing and refinement statistics.

Values in parentheses are for the outer resolution shell.

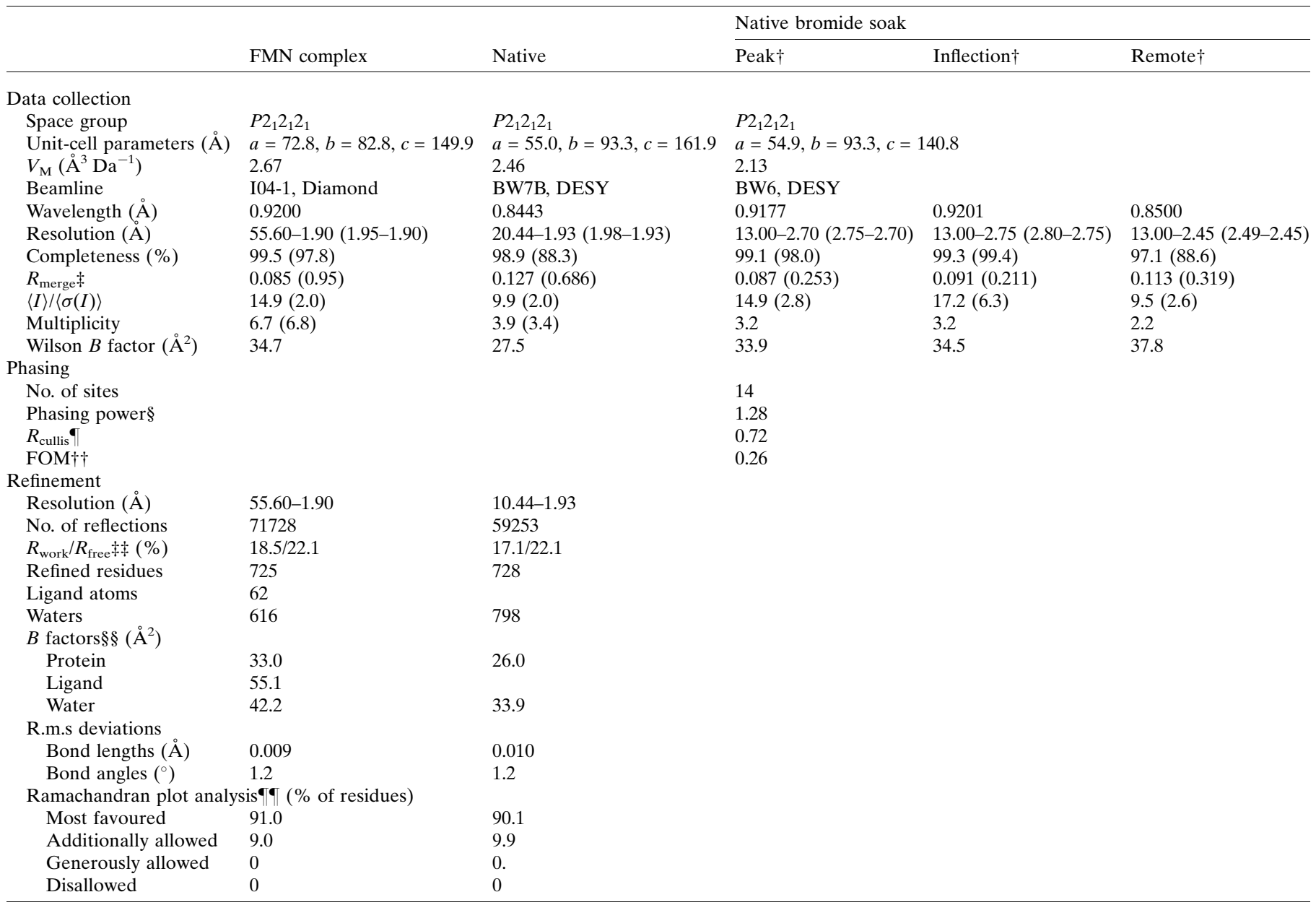

$\dagger I(+)$ and $I(-)$ were scaled separately for anomalous data. $\quad \ddagger R_{\text {merge }}=\sum_{h k l} \sum_{i}\left|I_{i}(h k l)-\langle I(h k l)\rangle\right| / \sum_{h k l} \sum_{i} I_{i}(h k l)$, where $I(h k l)$ is the intensity of reflection $h k l, \sum_{h k l}$ is the sum over all reflections and $\sum_{i}$ is the sum over $i$ measurements of the reflection. \& Phasing power $=F_{\mathrm{H}} / E$, where $E$ is the estimated lack-of-closure error. I $R_{\text {cullis }}=$ lack-of-closure error $/$ isomorphous difference and is quoted for centric reflections only. †† FOM is the overall figure of merit, defined as the estimated cosine of the phase error. $\$ \neq R_{\text {cryst }}=$ $\sum_{h k l}|| F_{\mathrm{obs}}|-| F_{\text {calc }}|| / \sum_{h k l}\left|F_{\mathrm{obs}}\right|$. $\S \S$ The Wilson B factor was estimated by SFCHECK (Vaguine et al., 1999). थ (Laskowski et al., 1993).

data were collected at $100 \mathrm{~K}$ at station BW6 at the Max Planck Institute at DESY, Hamburg, Germany (Table 1) using a MAR165 CCD detector (MAR Research). The data were processed using the DENZO suite of programs (Otwinowski \& Minor, 1997).

Data for the complex of overexpressed 3,6-DKMO with FMN were collected at $100 \mathrm{~K}$ on beamline I04-1 at the Diamond Synchrotron light source (Oxford, England) using a PILATUS detector (Dectris, Baden, Switzerland). The complex data were processed and the native apo data were reprocessed using XDS (Kabsch, 2010) in the xia2 (Winter et $a l ., 2013$ ) pipeline. Further data and model manipulation was carried out using the $C C P 4$ suite of programs (Winn et al., 2011).

\subsection{Structure determination}

The structure of native apo 3,6-DKMO was solved by a combination of MR, Br-soak MAD and density modification, as previously described in Isupov \& Lebedev (2008). MR trials were performed using a synthetic $\alpha_{2}$ dimer of the bacterial luciferase from Vibrio harveyi (16\% sequence identity; Fisher et al., 1996). An exhaustive translation search in MOLREP (Vagin \& Teplyakov, 2010) was conducted for all possible orientations of the dimeric polyalanine model (Lebedev et al., 2008) in which the orientation of its molecular dyad coincided with orientation of the noncrystallographic twofold axis determined from the self-rotation function at $\varphi=78.4^{\circ}, \psi=$ $40.8^{\circ}, \chi=180.0^{\circ}$. A convincing translation-function solution for a single orientation was subjected to rigid-body refinement in MOLREP (Vagin et al., 1998) and positional refinement in REFMAC5 (Murshudov et al., 2011). The $23 \mathrm{Br}$ sites were located in the anomalous difference Fourier synthesis using phases obtained by multi-crystal averaging with DMMULTI (Cowtan, 2010) and were used in MAD phasing with MLPHARE (Otwinowski, 1991). While anomalous substructure search programs such as SHELXD (Sheldrick, 2010) can 
often find the $\mathrm{Br}$ sites for structures of this size (see, for example, Vivoli et al., 2014), the sites in the 3,6-DKMO anomalous data could not be located, probably owing to a weak signal caused by the higher mosaicity of the soaked crystals.

The phases from DMMULTI averaging were used in REFMAC5 phased refinement (Pannu et al., 1998) to produce a good-quality map that allowed the building of an apo 3,6DKMO atomic model in $O$ (Jones et al., 1991).

The structure of the FMN complex of the overexpressed 3,6-DKMO was solved by MR with the refined structure of the apoenzyme using MOLREP (Vagin \& Teplyakov, 2010). The structure was refined by REFMAC5 and the model was rebuilt in Coot (Emsley et al., 2010). BUSTER refinement (Bricogne et al., 2015) was used for better positioning of the cofactor with partial occupancy in the enzyme active site. The figures were prepared using PyMOL (DeLano, 2002) and CCP4mg (McNicholas et al., 2011).

\section{Results and discussion}

\subsection{Purification and crystallization}

The native 3,6-DKMO crystallized in space group $P 2{ }_{1} 2_{1} 2_{1}$, with unit-cell parameters $a=55.0, b=93.4, c=162.0 \AA$. The crystals that were grown in $50 \mathrm{~m} M$ PIPES pH 6.5, $50 \%$ ammonium sulfate (McGhie et al., 1998) contained two DKMO subunits in the asymmetric unit with a solvent content of $50 \%$. The crystal of native protein used for $\mathrm{Br}$ anomalous phasing and for multi-crystal averaging belonged to the same space group, with the different unit-cell parameters $a=54.9$, $b=93.3, c=140.8 \AA$ and with $42 \%$ solvent content. The use of the Takara overexpression system was successful in producing a good yield of soluble 3,6-DKMO protein in E. coli host cells. The protein was purified by nickel-affinity and gel-filtration chromatography. The best overexpressed protein crystals grew at $18^{\circ} \mathrm{C}$ from $100 \mathrm{~m} M$ HEPES $\mathrm{pH} 7.0,20 \%$ PEG 3350 in the presence of $20 \mathrm{~m} M$ FMN, $5 \mathrm{~m} M$ NADH and $5 \mathrm{~m} M(-)$ camphor. These crystals contained a dimeric 3,6-DKMO molecule in the asymmetric unit and belonged to space group $P 2{ }_{1} 2_{1} 2_{1}$, with unit-cell parameters $a=72.8, b=82.8, c=149.9 \AA$ and with $54 \%$ solvent content.

\subsection{Quality of the models}

The native apo structure was refined to an $R_{\text {cryst }}$ and $R_{\text {free }}$ of 17.1 and $22.1 \%$, respectively, for all data in the resolution range $20-1.9 \AA$ without a $\sigma$ cutoff (Table 1 ). The FMN complex was refined to an $R_{\text {cryst }}$ and $R_{\text {free }}$ of 18.5 and $22.1 \%$, respectively, at $75-1.9 \AA$ resolution. The following residues were not modelled owing to disorder: $A 1, A 128-133, A 176-$ 180, $A 377-378, B 1, B 127-135$ and $B 176-179$ in the native structure and $A 126-135, A 176-179, A 377-378, B 125-135$ and $B 176-179$ in the FMN-complex structure.

The native 3,6-DKMO model also contains 798 water molecules, several glycerol molecules, four sulfate ions and two PIPES buffer molecules. The FMN-complex structure contains two cofactor molecules, with the occupancy of the cofactor modelled at 0.6 , and several polyethylene glycol molecules of variable length. The two subunits of the native structure were rebuilt independently. The root-mean-square deviation (r.m.s.d.) between $\mathrm{C}^{\alpha}$ positions of the two subunits is $0.23 \AA$. The FMN complex structure has been refined with local NCS restraints implemented in REFMAC5 (Murshudov et al., 2011). The r.m.s.d. between the $\mathrm{C}^{\alpha}$ positions of the two subunits is $0.19 \AA$. Both models contain no Ramachandran outliers as identified by PROCHECK (Laskowski et al., 1993). The overall $G$-factors used as a measure of the stereochemical quality of the model are 0.2 for the native structure and 0.1 for the FMN complex (PROCHECK) and are better than expected for the reported resolution. A total of 80 side chains in the native structure and 92 in the FMN complex structure were modelled with alternative conformations. The residues Pro11 and Ala77 are in the cis conformation. Approximately $43 \%$ of the $3,6-\mathrm{DKMO}$ amino acids are in $\alpha$-helices, $14 \%$ in $\beta$-sheets and $5 \%$ in $33_{10}$-helices.

\subsection{Overall fold}

The 3,6-DKMO monomer is made up from a single domain with an eight-stranded $\alpha / \beta$-barrel (TIM-barrel) fold which has the nonprolyl cis-residue Ala77 forming a $\beta$-bulge in the middle of $\beta$-strand 3. The TIM-barrel structure (Banner et al., 1976; Wierenga, 2001) is one of the most common protein folds, which can accommodate a variety of functions. Interestingly, according to the SCOP database (Andreeva et al., 2014), these include at least two superfamilies of FMNdependent oxygenases. These are the bacterial luciferase-like superfamily to which DKMOs belong (for example, luciferase from $V$. harveyi; PDB entry 1luc; Fisher et al., 1996) and the FMN-linked oxidoreductase superfamily (for example, old yellow enzyme from Thermus scotoductus SA-01; PDB entry 3hgj; Opperman et al., 2010). The 3,6-DKMO enzyme described in this paper contains two additional $\beta$-sheets: a hairpin formed by residues $159-162$ and $167-170$ and a parallel two-stranded $\beta$-sheet formed by residues 259-261 and 320-322 (Fig. 2a).

Two 3,6-DKMO subunits join to form a dimeric molecule (Fig. 2b) where approximately $3300 \AA^{2}$ of solvent-accessible area is buried upon dimer formation (18\% of the solventaccessible area of a subunit).

\subsection{FMN binding to DKMO}

In the course of the purification of the 3,6-DKMO enzyme the cofactor FMN is easily lost, especially if an ammonium sulfate precipitation step is used in the purification procedure, since the inorganic sulfate competes for the cofactor-binding site. We attribute the low affinity of the enzyme for the oxidized form of the cofactor to be owing to the catalytic cycle of DKMO. The enzyme is likely to have a higher affinity for the reduced form of the cofactor $\mathrm{FMNH}_{2}$ (FNR), which has a bent conformation in the isoalloxazine ring. Similarly, a 200fold higher affinity for FNR compared with FMN has been reported for another two-component $p$-hydroxyphenylacetate monooxygenase from Acinetobacter baumannii (Sucharitakul 


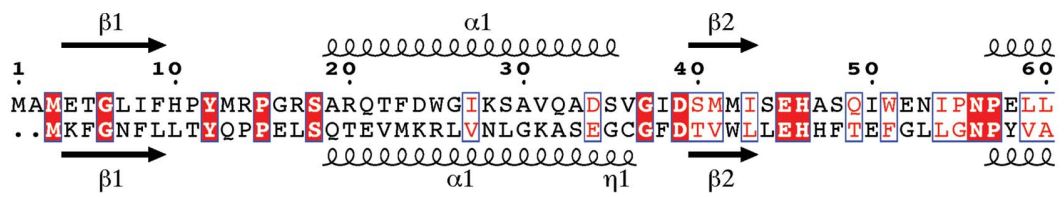

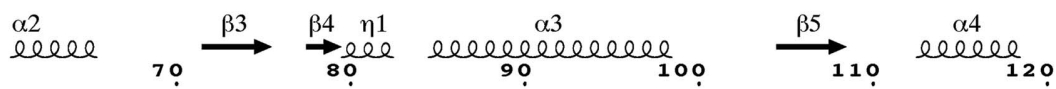

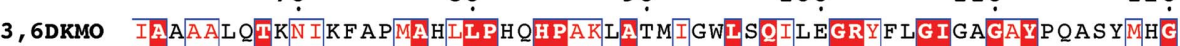
LucA_VH AAHLLGATETLNVGTAAIVLPTAH PVRQAEDVNLIDQMS KGRFRFG ICRGLYDKDFRVFG

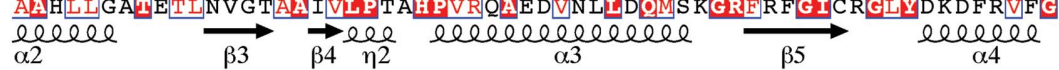

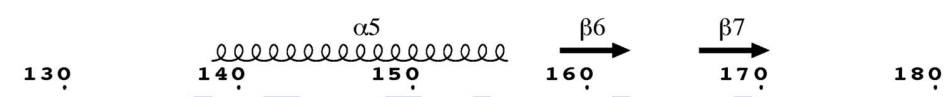
3,6DKMO IRNAGQSNTATGGEETKNLNDMVRESLFIMEKI同KREPFFH ITKYWDAGYPEELEGEEGD

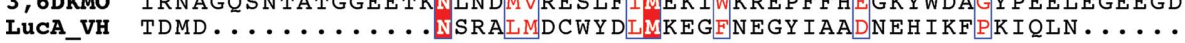

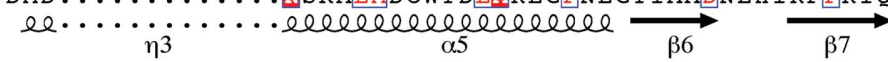

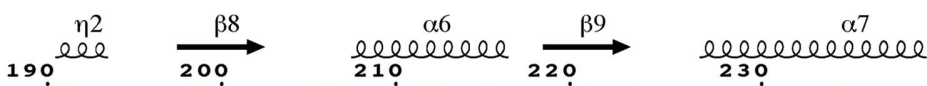
3,6DKMO EQHKLADFSP同GGKAPETAV̈TGFSY NSP SMRLAGERNFKPVISIFS . GLDALKRHWEVYSE LuCA_VH .......PSAYTQGGAPVYVVAE..SASTTEWAAERGLPMILSWI INTHE KKAQLDLYNE $\underset{\beta 8}{\longrightarrow} \underset{\alpha 6}{\longrightarrow} \underset{\beta 9}{\ln } \underset{\alpha 7}{\longrightarrow}$

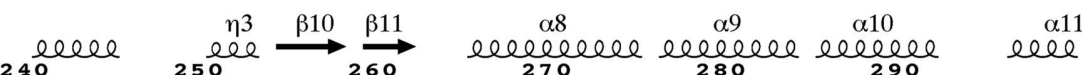
3,6DKMO 240 AंAIFAGHTPDRSRHAVSHTVFCADTDKEAKRLVMEGPIGYCFERYLIPIWRRFGMMDGYA LUCA_VH VATEHGYDVTKIDHCLSYITSVDHDSNR................ RNFIGHWYDSYVNATKI leele ele $\underset{\beta 10}{\rightarrow} \underset{\beta 11}{\rightarrow}$ ele.......elel.elebelelelel

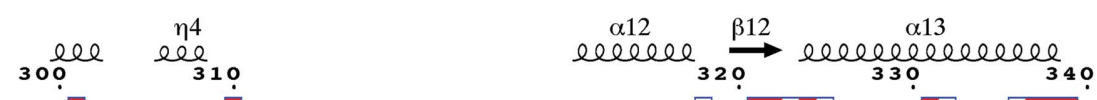
3,6DKMO KDAGIDPVDAD. .................... LUCA_VH FDDSDQTKGYDFNKGQWRDFVLKGH KDTNRRIDYSYEIN PVGTPEICIAIIOQDIDATGI ele

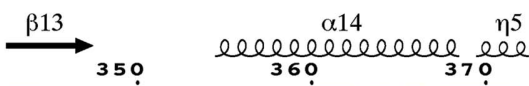

3, 6DKMO WGTLOVEAHDYY DDPAPWFOSLELISKEVAPKILLPKR LUCA_VH D.NICCGFEANGS.EEEIIASMKLFO SDVMPTLKEKO $\underset{\beta 13}{\longrightarrow}$ el ${ }_{7}$ lebelebelel ele

(a)

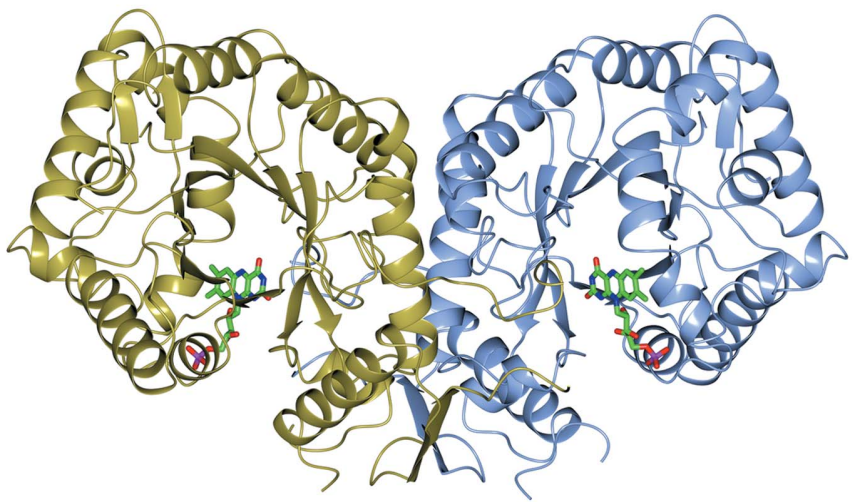

Figure 2

(b)

(a) Amino-acid sequence alignment of 3,6-DKMO with the $\alpha$ subunit of $V$. harveyi luciferase. The secondary-structure elements of 3,6-DKMO and luciferase are indicated above and below the alignment, respectively, as $\alpha$-helices, $\eta$-helices $\left(3_{10}\right.$-helices) and $\beta$-strands. The secondary-structure assignment and the figure were produced using ESPript (Robert \& Gouet, 2014). (b) A cartoon representation of the dimeric molecule of 3,6-DKMO showing its classical $\alpha / \beta$-barrel structure. The FMN cofactor is shown as a stick model. Figs. $2(b), 4(b)$ and 5 were prepared using $C C P 4 m g$ (McNicholas et al., 2011) et al., 2006). The planar FMN, which has low affinity for the 3,6-DKMO active site, leaves it to be reduced to FNR by the FMN reductase. As the FMN reductase from the CAM plasmid could not be purified in significant amounts from $P$. putida preparations, commercially available NADH-FMN oxidoreductase from $V$. harveyi has successfully been used for reduction of the cofactor in activity measurements (McGhie, 1998). More recently, an E. coli flavin oxidoreductase was used to form a functional assembly with type II DKMOs (Kadow et al., 2014).

\subsection{FMN cofactor modelling in the electron density}

When the structures of unligated 3,6DKMO and the $\alpha$ subunit of the holo bacterial luciferase (PDB entry 3fgc; Campbell et al., 2009) are superimposed, the FMN ring of luciferase has steric clashes with the active-site residues of DKMO. It appears that either the isoalloxazine ring binds differently in the DKMO active site or some conformational changes need to occur to accommodate the FMN molecule in a similar orientation.

Multiple attempts to co-crystallize native DKMO and later the overexpressed protein with increasing concentrations of FMN were not very successful, as the low affinity of the oxidized cofactor for the 3,6-DKMO enzyme makes it difficult to achieve cofactor binding. No conformational changes were observed in the active site of FMN-complexed 3,6-DKMO, which is in agreement with the rigidity of the TIM-barrel scaffold. The best density for the cofactor molecule was achieved when the protein was co-crystallized in PEG with a large excess $(20 \mathrm{mM})$ of FMN, the substrate analogue (-)camphor and NADH. Attempts to use higher concentrations of cofactor resulted in the appearance of FMN crystals in the crystallization wells. A similarly poor affinity of the cofactor for the active site was observed in other reported structures of the bacterial luciferase-like fold superfamily enzymes which have either partial occupancy of FMN as in the $V$. harveyi luciferase 
(Campbell et al., 2009) or have $B$ factors which are significantly higher than those of neighbouring residues as observed in the structures of nitrilotriacetate monooxygenase from Bacillus subtilis and long-chain alkane monooxygenase from Geobacillus thermodenitrificans (PDB entries 1yw1 and 3b9o; Bonanno et al., 2005; Li et al., 2008).

Therefore, detailed modelling of the position of the FMN cofactor in the 3,6-DKMO active site was carried out. When the native 3,6-DKMO model was positioned in the complex unit cell and refined by BUSTER (Bricogne et al., 2015) prior to thee addition of solvent molecules, the weighted $F_{\mathrm{o}}-F_{\mathrm{c}}$ electron-density maps clearly indicated the presence of the cofactor and the position of its phosphate group; however, the density for the isoalloxazine ring and the density for the ribityl sugar were less well defined (Fig. 3). The electron density clearly identifies the plane of the cofactor ring, with N5 of FMN hydrogen-bonded to His10. The cofactor atoms N3, O2 and $\mathrm{O} 4$ form hydrogen bonds to the 3,6-DKMO protein residues in this orientation. The isoalloxazine ring could also be modelled by a rotation of $180^{\circ}$ around the $\mathrm{C1}^{\prime}-\mathrm{N} 1$ bond; however, in this case the $\mathrm{C} 7 \mathrm{M}$ and $\mathrm{C} 8 \mathrm{M} \mathrm{C}$ atoms were seen to badly clash with the protein residues. Subsequent refinement varying the partial occupancy of the cofactor has indicated that a cofactor occupancy of 0.6 gives the best fit to both the $2 F_{\mathrm{o}}-F_{\mathrm{c}}$ and $F_{\mathrm{o}}-F_{\mathrm{c}}$ maps.

The poor electron density for FMN observed in the active site of 3,6-DKMO could indicate disorder related to the existence of multiple cofactor species created by photoreduction in the intense synchrotron X-ray beam. This has previously been reported for other flavin cofactor-containing enzymes (Røhr et al., 2010). However, the density for the isoalloxazine ring of the cofactor in 3,6-DKMO appears to be flat and not butterfly-shaped as would be observed for the reduced species. We therefore consider the effect of photoreduction to be insignificant and attribute the poor electron density to the low affinity of the FMN for the active site of the enzyme.

This observation is supported by our experience with the structure of a related protein (Isupov \& Littlechild, unpublished data), determined at $1.7 \AA$ resolution, in which the FMN is at full occupancy in the active site. In this case the clear electron density shows that the FMN isoalloxazine ring is flat, thereby confirming the prevalence of the oxidized species. This reinforces our interpretation as described above for the 3,6-DKMO since crystals of both enzymes were subjected to a comparable radiation exposure.

No electron density was observed for the (-)-camphor or the NADH present in the crystallization medium.

\subsection{The active site}

The FMN cofactor binds in the cleft formed at the C-terminal side of the TIM barrel (Fig. $2 b$ ), with the phosphate group involved in hydrogen bonding at the edge of the cleft and the isoalloxazine ring located deep inside of the cleft (Figs. $4 a$ and $4 b$ ). The adjacent subunit contributes residues 175-180 to the lining of the active-site cavity on the opposite side of the cleft. This loop is poorly ordered in both the apo and the complex structures. The isoalloxazine ring makes hydrogen bonds to the side chains of His10 and Ser44 and the main-chain $\mathrm{O}$ atom of Met76.

The reduced form of the cofactor FMN (FNR) model was created using JLigand (Lebedev et al., 2012), with the FNR
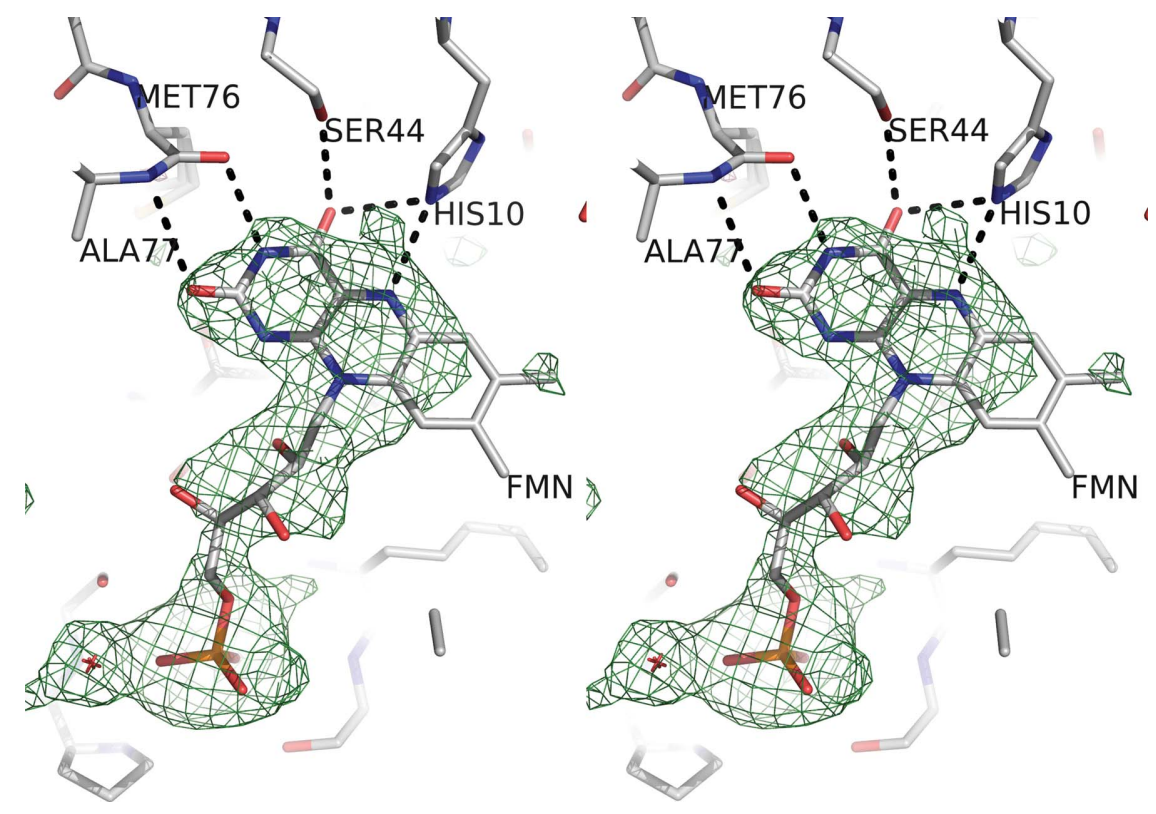

Figure 3

A stereo diagram of the active site of 3,6-DKMO viewed from the solvent region. The cofactor FMN, modelled with an occupancy of 0.6, and neighbouring residues are shown as stick models. The positive OMIT $F_{\mathrm{o}}-F_{\mathrm{c}}$ electron-density map contoured at $3.0 \sigma$ is shown in green and the negative $F_{\mathrm{o}}-F_{\mathrm{c}}$ map contoured at $3.0 \sigma$ is shown in red. The OMIT difference electron density was calculated by BUSTER using a structure from which all solvent and cofactor molecules had been removed and the model refined to reduce the bias. The figure was prepared using PyMOL (DeLano, 2002). 
isoalloxazine ring modelled with a $20^{\circ}$ deviation from planarity, as observed in the structure of another luciferase-

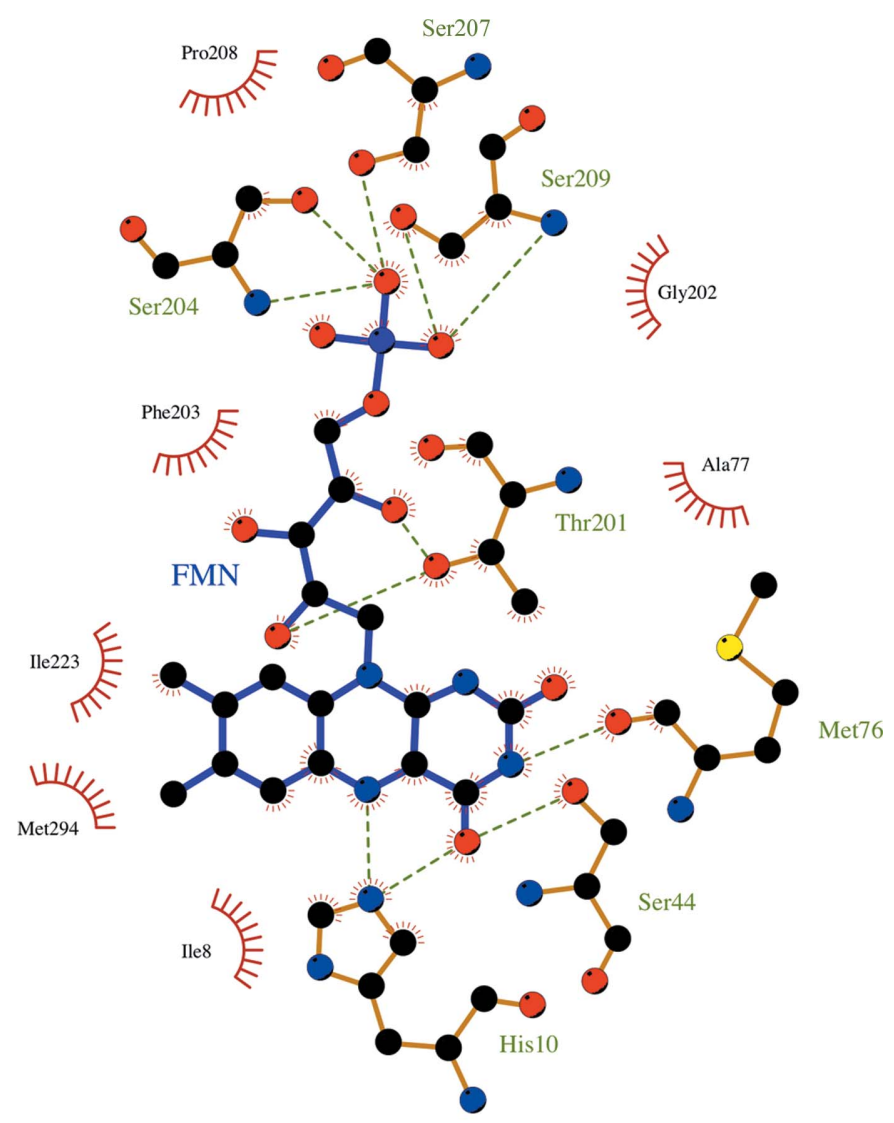

(a)

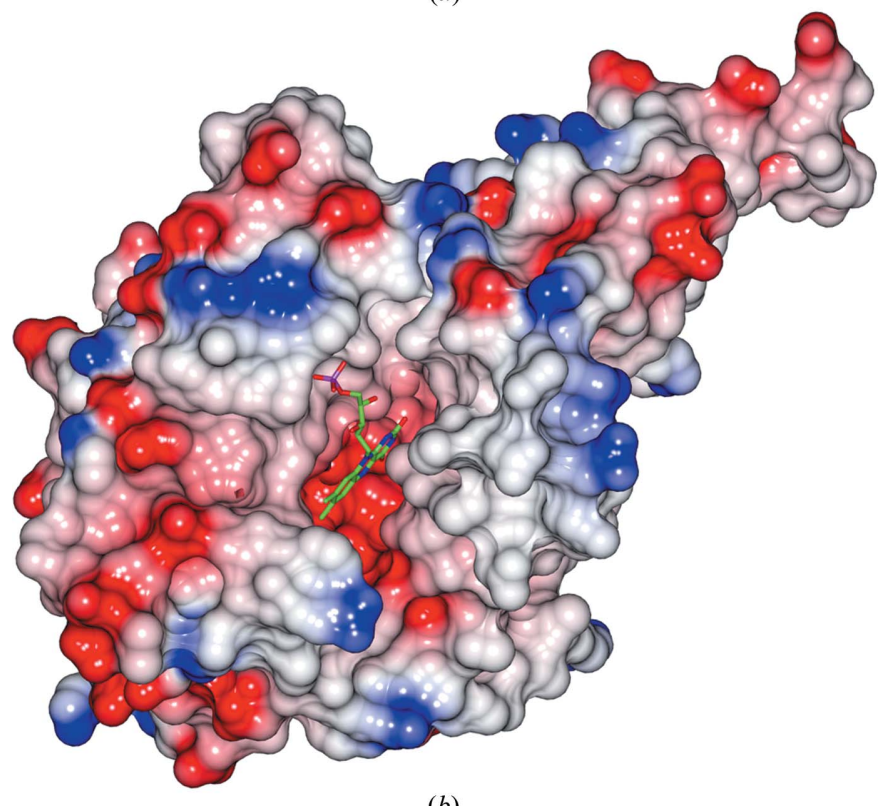

(b)

Figure 4

(a) A schematic drawing of the interactions of the FMN in the active site of 3,6-DKMO. The figure was prepared by LigPlot+ (Laskowski \& Swindells, 2011). (b) The electrostatic potential surface calculated for the subunit of 3,6-DKMO. Positive charge is shown in blue and negative charge in red. The active-site cavity can be seen in the centre of the figure. The cofactor FMN molecule is shown as a stick model at the bottom of the active-site pocket. like fold superfamily enzyme, the $\mathrm{F}_{420}$-dependent secondary alcohol dehydrogenase enzyme (PDB entry 1rhc; Aufhammer et al., 2004), and several other reduced flavin cofactorcontaining structures. The FNR model was positioned into the active site of 3,6-DKMO, where it appears to retain the hydrogen bonding observed for the oxidized FMN (Fig. 4a) but makes additional favourable hydrophobic interactions with the side chains of Phe203 and Ile223. These interactions would favour the higher affinity of the enzyme for the reduced form of the cofactor.

The catalytic reaction occurs on the si side of the isoalloxazine ring, which is consistent with the mechanism of other bacterial luciferase-like fold enzymes, but contradicts the predictions previously made for the $P$. putida type II BVMOs (Kelly, 1996).

\subsection{Comparison with other bacterial luciferase-family proteins}

The dimer structure is conserved throughout the bacterial luciferase-like superfamily of TIM-barrel proteins and its conservation was used for macromolecular phasing of 3,6DKMO (Isupov \& Lebedev, 2008). Enzymes belonging to the other superfamily of TIM-barrel fold FMN-linked oxidoreductases also form dimers and occasionally higher oligomers, but these dimers are organized differently.

The closest structural homologue to 3,6-DKMO among known structures is subunit $A$ of $V$. harveyi luciferase (PDB entry 1luc; Fisher et al., 1996), which can be superimposed with the 3,6-DKMO structure with an r.m.s.d. of $2.2 \AA$ for $292 \mathrm{C}^{\alpha}$ atoms of 362, as calculated by Coot (Emsley et al., 2010). Superimposition of the 3,6-DKMO enzyme monomer $\mathrm{C}^{\alpha}$ positions with the FMN-dependent long-chain alkane monooxygenase (PDB entry 3b9o; Li et al., 2008) over 299 residues gives an estimated r.m.s.d. of $2.6 \AA$. The subunit of the $\mathrm{F}_{420^{-}}$ dependent secondary dehydrogenase enzyme (PDB entry

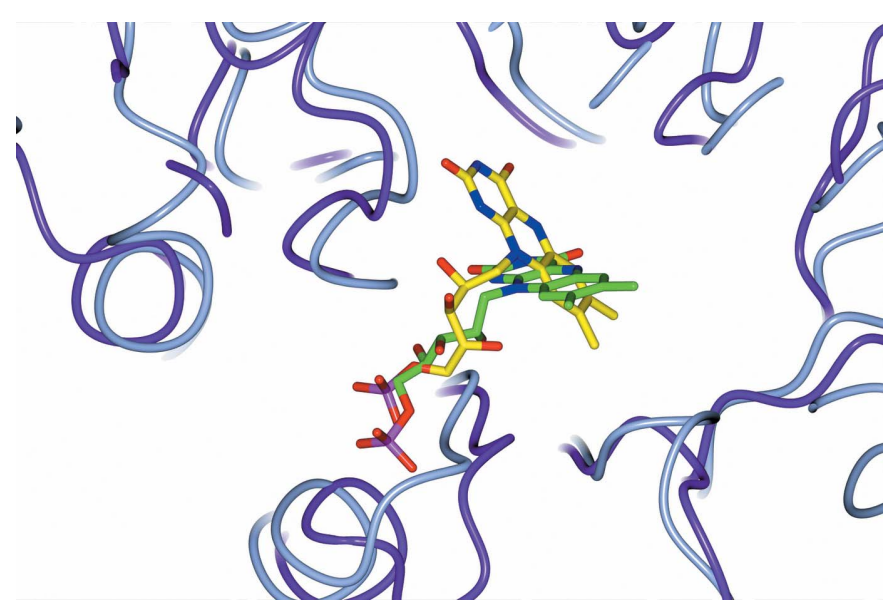

Figure 5

A diagram showing the different orientation of the isoalloxazine ring of the FMN cofactor in the active sites of the superimposed $\alpha$ subunit of the bacterial luciferase and the 3,6-DKMO enzyme. The $\mathrm{C}^{\alpha}$ backbones of luciferase and 3,6-DKMO are shown in ice blue and purple, respectively. The $\mathrm{C}$ atoms in the FMN cofactors are shown in yellow and green for luciferase and 3,6-DKMO, respectively. 
1rhc; Aufhammer et al., 2004) can be superimposed with an r.m.s.d. of $2.8 \AA$ over 280 residues.

Known structures of FMN- and $\mathrm{F}_{420}$-dependent enzymes have a consistent orientation of their tricyclic cofactor rings within the clefts of their active sites (Aufhammer et al., 2004, 2005; Bonanno et al., 2005; Li et al., 2008; Campbell et al., 2009). The orientation of the isoalloxazine ring in the active site of 3,6-DKMO is nearly normal to the planes of the tricyclic cofactor rings observed in known structures of the bacterial luciferase-like fold superfamily enzymes, as illustrated in Fig. 5 for the bacterial luciferase $\alpha$ subunit. Despite this difference in the orientation of the cofactor ring, all enzymes of the bacterial luciferase superfamily catalyse their reaction on the si side of the ring.

\subsection{The reaction mechanism}

The catalytic activity of the type II BMVOs is carried out mainly through the prosthetic FMN group. This cofactor binds in the enzyme active site in the FNR form, which undergoes attack by molecular oxygen at $\mathrm{C} 4 \mathrm{~A}$ of the isoalloxazine ring and becomes $4 \alpha$-hydroperoxyflavin (FMN-OOH). This reaction is similar to that described for the flavin-containing monooxygenase from Schizosaccharomyces pombe (Eswaramoorthy et al., 2006). The active-site residues are required for positioning of the substrate in a catalytic position in relation to the cofactor. Since there is a variation of the position of the cofactor isoalloxazine ring between 3,6-DKMO and the other proteins of the bacterial luciferase family, there is little conservation of the active-site residues within this enzyme family. Even the closely related 3,6- and 2,5-DKMOs have only two conserved residues lining the active-site cavity, which are His46 and Glu52 in 3,6-DKMO numbering. These are not conserved within the protein family. The residues His10 and Ser44 which are involved in FMN isoalloxazine ring binding in 3,6-DKMO are not conserved in 2,5-DKMO, implying a different mode of cofactor binding in this related enzyme.

\subsection{Nonprolyl cis-peptide $\beta$-bulge}

A common feature of many bacterial luciferase-like fold superfamily enzymes is a nonprolyl cis-peptide forming a bulge in the middle of $\beta 3$ which was proposed to be important for catalytic activity (Aufhammer et al., 2005). This feature is not present in the nitrilotriacetate monooxygenase and long-chain alkane monooxygenase (Bonanno et al., 2005; Li et al., 2008). The importance of the nonprolyl cis-peptide $\beta$-bulge seems to be confirmed in the 3,6-DKMO structure, where this bulge is formed by residues Met76 and cis Ala77, with the main-chain $\mathrm{O}$ atom of Met76 and $\mathrm{N}$ atom of Ala77 contributing to the binding of the FMN ring.

\subsection{Interaction with the flavin reductase}

The FMN reductase activity is required for the 3,6-DKMO reaction. When native 3,6-DKMO was purified from camphorinduced cells of $P$. putida, high enzymatic activity was observed in the crude extract (McGhie \& Littlechild, 1996; McGhie et al., 1998). This activity was present for the partially purified enzyme; however, it was lost in the later stages of purification of the oxygenating component. This led to the conclusion (McGhie et al., 1998) that the oxygenating component forms a transient multienzyme complex with a specific $P$. putida flavin reductase. The commercially available Vibrio flavin reductase (Sigma-Aldrich) was able to demonstrate activity with the purified 3,6-DKMO oxygenating enzyme in biotransformation reactions (McGhie, 1998). More recently, it has been reported (Kadow et al., 2014) that one of the flavin reductases present in E. coli is able to catalyse the FMN reduction with both 3,6- and 2,5-DKMOs. Partial sequencing of the large CAM plasmid has now identified a flavin reductase adjacent to the 3,6-DKMO gene on the CAM plasmid (Littlechild \& Isupov, unpublished data), and studies are ongoing to reconstitute the putative multienzyme complex in a stable form to allow its structural characterization.

\section{Acknowledgements}

The authors would like to thank Dr Paul James and Dr Mirella Vivoli for useful discussions. The authors would like to thank EMBL Hamburg for access to DESY beamline BW7B, Max Planck Institute Hamburg for access to beamline BW6 and Diamond Light Source for access to beamline I04-1 (proposal No. MX8889) and the beamline staff scientists. We acknowledge support under the European Community Access to Research Infrastructure Action of the Improving Human Potential Programme to EMBL Hamburg Outstation, contract No. HPRI-CT-1999-00017. MNI is grateful to the University of Exeter and BBSRC-funded ERA-IB grant BB/L002035/1. The BBSRC are thanked for the award of grant No. BB/D004403/1 and earlier grants including a BBSRC PhD studentship to EJM. The work was also supported by a large consortium EU Framework 4 grant on 'Biotransformations using BaeyerVilliger Monooxygenases'. MNI carried out the structural aspects of the project and contributed to the writing of this manuscript. ES, JB, RPG and CS were postdoctoral fellows in the Exeter group funded by BBSRC grants. EJM carried out the original characterization and crystallization of native DKMO. GD, VH, SAD and CFD were other students in the Exeter group who contributed to this work. PCL, YH and HI determined the initial sequence of the 3,6-DKMO enzyme. UTB, MK and KB provided the plasmids encoding the 3,6DKCMO gene and supported the work with regard to the overexpression and purification protocols. GB helped to collect data from the bromide-soaked crystal, which contributed to the structural determination of the native enzyme. JAL coordinated the project and wrote the manuscript with MNI.

\section{References}

Alphand, V., Carrea, G., Wohlgemuth, R., Furstoss, R. \& Woodley, J. M. (2003). Trends Biotechnol. 21, 318-323.

Andreeva, A., Howorth, D., Chothia, C., Kulesha, E. \& Murzin, A. G. (2014). Nucleic Acids Res. 42, D310-D314.

Aufhammer, S. W., Warkentin, E., Berk, H., Shima, S., Thauer, R. K. \& Ermler, U. (2004). Structure, 12, 361-370.

Aufhammer, S. W., Warkentin, E., Ermler, U., Hagemeier, C. H., Thauer, R. K. \& Shima, S. (2005). Protein Sci. 14, 1840-1849. 
Baeyer, A. \& Villiger, V. (1899). Ber. Dtsch. Chem. Ges. 32, 36253633.

Balke, K., Kadow, M., Mallin, H., Sass, S. \& Bornscheuer, U. T. (2012). Org. Biomol. Chem. 10, 6249-6265.

Banner, D. W., Bloomer, A. C., Petsko, G. A., Phillips, D. C. \& Wilson, I. A. (1976). Biochem. Biophys. Res. Commun. 72, 146-155.

Beecher, J. \& Willetts, A. (1998). Tetrahedron Asymmetry, 9, 18991916.

Bonanno, J. B. et al. (2005). J. Struct. Funct. Genomics, 6, 225-232.

Bornscheuer, U. T., Huisman, G., Kazlauskas, R. J., Lutz, S., Moore, J. \& Robins, K. (2012). Nature (London), 485, 185-194.

Bricogne, G., Blanc, E., Brandl, M., Flensburg, C., Keller, P., Paciorek, P., Roversi, P., Sharff, A., Smart, O., Vonrhein, C. \& Womack, T. (2015). BUSTER v.2.10.2. Cambridge: Global Phasing Ltd.

Campbell, Z. T., Weichsel, A., Montfort, W. R. \& Baldwin, T. O. (2009). Biochemistry, 48, 6085-6094.

Conrad, H. E., Dubus, R., Namtvedt, M. J. \& Gunsalus, I. C. (1965). J. Biol. Chem. 240, 495-503.

Cowtan, K. (2010). Acta Cryst. D66, 470-478.

Dauter, Z., Dauter, M. \& Rajashankar, K. R. (2000). Acta Cryst. D56, 232-237.

DeLano, W. L. (2002). PyMOL. http://www.pymol.org.

Emsley, P., Lohkamp, B., Scott, W. G. \& Cowtan, K. (2010). Acta Cryst. D66, 486-501.

Eswaramoorthy, S., Bonanno, J. B., Burley, S. K. \& Swaminathan, S. (2006). Proc. Natl Acad. Sci. USA, 103, 9832-9837.

Fisher, A. J., Thompson, T. B., Thoden, J. B., Baldwin, T. O. \& Rayment, I. (1996). J. Biol. Chem. 271, 21956-21968.

Gagnon, R., Grogan, G., Levitt, M. S., Roberts, S. M., Wan, P. W. H. \& Willetts, A. J. (1994). J. Chem. Soc. Perkin Trans. 1, pp. 25372543.

Gagnon, R., Grogan, G., Roberts, S. M., Villa, R. \& Willetts, A. J. (1995). J. Chem. Soc. Perkin Trans. 1, pp. 1505-1511.

Grogan, G., Roberts, S., Wan, P. \& Willetts, A. J. (1993). Biotechnol. Lett. 15, 913-918.

Grogan, G., Roberts, S., Wan, P. \& Willetts, A. (1994). Biotechnol. Lett. 16, 1173-1178.

Grogan, G., Roberts, S. M. \& Willetts, A. J. (1992). Biotechnol. Lett. 14, 1125-1130.

Grogan, G., Roberts, S. \& Willetts, A. J. (1993). J. Chem. Soc. Chem. Commun., pp. 699-701.

Isupov, M. N. \& Lebedev, A. A. (2008). Acta Cryst. D64, 90-98.

Jones, K. H., Smith, R. T. \& Trudgill, P. W. (1993). J. Gen. Microbiol. 139, 797-805.

Jones, T. A., Zou, J.-Y., Cowan, S. W. \& Kjeldgaard, M. (1991). Acta Cryst. A47, 110-119.

Kabsch, W. (2010). Acta Cryst. D66, 125-132.

Kadow, M., Balke, K., Willetts, A., Bornscheuer, U. T. \& Bäckvall, J. E. (2014). Appl. Microbiol. Biotechnol. 98, 3975-3986.

Kadow, M., Loschinski, K., Sass, S., Schmidt, M. \& Bornscheuer, U. T. (2012). Appl. Microbiol. Biotechnol. 96, 419-429.

Kelly, D. R. (1996). Tetrahedron Asymmetry, 7, 1149-1152.

Krow, G. R. (1993). Org. React. 43, 251-798.

Laskowski, R. A., MacArthur, M. W., Moss, D. S. \& Thornton, J. M. (1993). J. Appl. Cryst. 26, 283-291.

Laskowski, R. A. \& Swindells, M. B. (2011). J. Chem. Inf. Model. 51, 2778-2786.

Lebedev, A. A., Vagin, A. A. \& Murshudov, G. N. (2008). Acta Cryst. D64, 33-39.
Lebedev, A. A., Young, P., Isupov, M. N., Moroz, O. V., Vagin, A. A. \& Murshudov, G. N. (2012). Acta Cryst. D68, 431-440.

LeGall, J., Bertland, A. U., Namtvedt, M. J. \& Conrad, H. E. (1963). Fed. Proc. 22, 295.

Leisch, H., Morley, K. \& Lau, P. C. K. (2011). Chem. Rev. 111, 4165 4222.

Li, L., Liu, X., Yang, W., Xu, F., Wang, W., Feng, L., Bartlam, M., Wang, L. \& Rao, Z. (2008). J. Mol. Biol. 376, 453-465.

Lilly, M. D., Chauhan, R., French, C., Gyamerah, M., Hobbs, G. R., Humphrey, A., Isupov, M., Littlechild, J. A., Mitra, R. K., Morris, K. G., Rupprecht, M., Turner, N. J., Ward, J. M., Willetts, A. J. \& Woodley, J. (1996). Ann. N. Y. Acad. Sci. 782, 513-525.

Littlechild, J. A., Guy, J., Connelly, S., Mallett, L., Waddell, S., Rye, C. A., Line, K. \& Isupov, M. (2007). Biochem. Soc. Trans. 35, 15581563.

Malito, E., Alfieri, A., Fraaije, M. W. \& Mattevi, A. (2004). Proc. Natl Acad. Sci. USA, 101, 13157-13162.

McGhie, E. J. (1998). PhD Thesis, University of Exeter.

McGhie, E. J., Isupov, M. N., Schröder, E. \& Littlechild, J. A. (1998). Acta Cryst. D54, 1035-1038.

McGhie, E. \& Littlechild, J. (1996). Biochem. Soc. Trans. 24, 29 S.

McNicholas, S., Potterton, E., Wilson, K. S. \& Noble, M. E. M. (2011). Acta Cryst. D67, 386-394.

Murshudov, G. N., Skubák, P., Lebedev, A. A., Pannu, N. S., Steiner, R. A., Nicholls, R. A., Winn, M. D., Long, F. \& Vagin, A. A. (2011). Acta Cryst. D67, 355-367.

Opperman, D. J., Sewell, B. T., Litthauer, D., Isupov, M. N., Littlechild, J. A. \& van Heerden, E. (2010). Biochem. Biophys. Res. Commun. 393, 426-431.

Otwinowski, Z. (1991). Proceedings of the CCP4 Study Weekend. Isomorphous Replacement and Anomalous Scattering, edited by W. Wolf, P. R. Evans \& A. G. W. Leslie, pp. 80-86. Warrington: Daresbury Laboratory.

Otwinowski, Z. \& Minor, W. (1997). Methods Enzymol. 276, 307-326.

Pannu, N. S., Murshudov, G. N., Dodson, E. J. \& Read, R. J. (1998). Acta Cryst. D54, 1285-1294.

Reetz, M. T. (2013). J. Am. Chem. Soc. 135, 12480-12496.

Robert, X. \& Gouet, P. (2014). Nucleic Acids Res. 42, W320-W324.

Roberts, S. M. \& Wan, P. W. H. (1998). J. Mol. Catal. B Enzym. 4, 111-136.

Røhr, A. K., Åsmund, K., Hersleth, H. \& Andersson, K. K. (2010). Angew. Chem. Int. Ed. 49, 2324-2327.

Shaham, M., Chakrabarty, A. M. \& Gunsalus, I. C. (1973). J. Bacteriol. 116, 944-949.

Sheldrick, G. M. (2010). Acta Cryst. D66, 479-485.

Sucharitakul, J., Chaiyen, P., Entsch, B. \& Ballou, D. P. (2006). J. Biol. Chem. 281, 17044-17053.

Vagin, A. A., Murshudov, G. N. \& Strokopytov, B. V. (1998). J. Appl. Cryst. 31, 98-102.

Vagin, A. \& Teplyakov, A. (2010). Acta Cryst. D66, 22-25.

Vaguine, A. A., Richelle, J. \& Wodak, S. J. (1999). Acta Cryst. D55, 191-205.

Vivoli, M., Ayres, E., Beaumont, E., Isupov, M. N. \& Harmer, N. J. (2014). IUCrJ, 1, 28-38.

Wierenga, R. K. (2001). FEBS Lett. 492, 193-198.

Winn, M. D. et al. (2011). Acta Cryst. D67, 235-242.

Winter, G., Lobley, C. M. C. \& Prince, S. M. (2013). Acta Cryst. D69, 1260-1273.

Wohlgemuth, R. (2010). Curr. Opin. Biotechnol. 21, 713-724. 